\title{
Detection of possible factors favouring the evolution of migraine without aura into chronic migraine
}

\author{
G. C. Manzoni - L. L. Lombardi - S. Lana • \\ M. Maffezzoni $\cdot$ C. Camarda $\cdot$ P. Torelli
}

(C) Springer-Verlag 2012

\begin{abstract}
In a minority of cases, the natural history of migraine without aura (MO) is characterised over time by its evolution into a form of chronic migraine (CM). In order to detect the possible factors predicting this negative evolution of MO, we searched in our Headache Centre files for all clinical records that met the following criteria: (a) first visit between 1976 and 1998; (b) diagnosis of MO or of common migraine at the first observation, with or without association with other primary headache types; (c) $<15$ days per month of migraine at the first observation; and (d) at least one follow-up visit at least 10 years after the first visit. The patients thus identified were then divided into two groups based on a favourable/ steady evolution (Group A: $n=243,195$ women and 48 men) or an unfavourable evolution (Group B: $n=72,62$ women and 10 men) of their migraine over time. In the two groups, we compared various clinical parameters that were present at the first observation or emerged at the subsequent follow-up visits. The parameters that were statistically significantly more frequent in Group B-and can therefore be considered possible negative prognostic factors-were: (a) $\geq 10$ days per month of migraine at the first observation; (b) presence of depression at the first visit in males; and (c) onset of depression or arterial hypertension after the first observation but before transformation to CM in females. Based on these findings, in MO patients the high frequency of migraine attacks, comorbidity with depression, and the tendency to develop arterial
\end{abstract}

G. C. Manzoni $(\bowtie) \cdot$ L. L. Lombardi · S. Lana ·

M. Maffezzoni · P. Torelli

Headache Centre, University of Parma, Via Gramsci, 14,

43100 Parma, Italy

e-mail: giancamillo.manzoni@unipr.it

C. Camarda

University of Palermo, Palermo, Italy hypertension should require particular attention and careful management to prevent evolution into $\mathrm{CM}$.

Keywords Migraine $\cdot$ Migraine without aura $\cdot$ Chronic migraine $\cdot$ Chronic daily headache Chronic headache

\section{Introduction}

Mean age at onset of migraine without aura (MO) is about 20 years. Onset may occur as early as in childhood, but very rarely does over 50 . Around this age, most patients see that their migraine symptoms decrease and sometimes even resolve completely. In contrast, in a minority, but not negligible number, of patients, MO worsens over time, changing some of its previous clinical features and especially increasing in frequency until there are no more free intervals between attacks. Thus, MO is gradually transformed from a typical attack-like headache to a so-called chronic headache.

Several reports have been published on this subject in the literature. However, there is still no consensus of opinion on: (a) the type of headache that occurs in these patients; and (b) the factors that may favour MO worsening over time. We therefore tried to help clarify this issue by performing a review of the broad case series of patients followed over the years at our Headache Centre. We report here the preliminary data of our study. The final data will be presented in a future publication.

\section{Patients and methods}

We accessed the University of Parma Headache Centre files and took all clinical records of patients that met the 
following requirements: (a) first visit between 1976 and 1998; (b) diagnosis of MO or of common migraine at the first observation, with or without association with other primary headache types; (c) $<15$ days per month of migraine at the first observation; and (d) at least one follow-up visit at least 10 years after the first visit.

The 348 patients (286 women and 62 men) thus identified were divided into groups based on the diagnoses made at the subsequent visits, and particularly the diagnosis made during the most recent visit at the Centre. Diagnoses were established according to the criteria of the International Classification of Headache Disorders, 2nd Edition (ICHD-II) of 2004 [1] and its 2006 revision (ICHD-IIR) [2].

The MO patterns that the 348 patients exhibited over the years varied widely, not only from patient to patient, but also within the same patient showing a course of alternating improvement and worsening. For that reason, in order to compare patients with a steady or favourable evolution of MO over time to patients with an unfavourable evolution, we excluded 33 patients (29 women and four men) who over the years showed a cyclically fluctuating migraine pattern or developed a new form of headache that might not necessary be interpreted as an evolution of their original MO.

The remaining 315 patients (257 women and 58 men) were divided into two groups based on a steady/favourable evolution (Group A: $n=243,195$ women and 48 men) or an unfavourable evolution (Group B: $n=72,62$ women and 10 men) of their migraine over time. In each of the two groups we compared not only the clinical features of MO that were originally present at the first visit, but also several parameters related to the patients' family, physiological, past, and recent medical histories, both at the first visit and at the subsequent follow-up visits. Statistical analysis was performed using Student's $t$ test and the $\chi^{2}$ test.

\section{Results}

Mean age at the first observation of the 315 patients included in the study was $32.0 \pm 11.2$ years, with no significant differences between the 257 women (32.1 \pm 11.1 years $)$ and the 58 men $(31.6 \pm 11.9$ years $)$. In the 72 patients with an unfavourable MO evolution, mean age at the first observation was older (Group B: $35.3 \pm 11.6$ years), though in a non-statistically significant way, than in the 243 patients with a steady/favourable evolution (Group A: $30.5 \pm 11.1$ years). The difference was more marked in men $(38.7 \pm 8.8$ vs. $30.1 \pm 11.8)$ than in women $(34.8 \pm$ 11.9 vs. $30.6 \pm 10.9)$.

No statistically significant differences were found between Group A and Group B in age at the last observation ( $46.0 \pm 11.5$ and $52.2 \pm 12.5$, respectively), the number of years of observation (15.8 \pm 4.4 and $15.0 \pm 4.8)$, and the number of follow-up visits $(3.8 \pm 3.6$ and $5.0 \pm 4.8)$.

No significant differences were found between the two groups in age at onset of MO, family history of migraine, pain location, and duration of attacks at the time of the first observation.

If we exclude three patients in Group A (two women and one man) with a highly variable number of MO days per month and 12 patients-eight in Group A (seven women and one man) and four in Group B (three women and one man) - with no certain data in their clinical records about the number of MO days per month, the remaining 232 patients in Group A (186 women and 46 men) and the remaining 68 patients in Group B (59 women and nine men) showed a statistically significant difference in the number of MO days per month at the time of the first observation (Table 1).

In the patients' medical histories reported at the first observation, depression was more statistically significantly present $(p<0.05)$ in Group B men $(6 / 9$ corresponding to $66.7 \%$ ) than in Group A men (10/48 corresponding to $20.8 \%$ ).

Among the diseases that were reported at the subsequent visits but occurred before MO "chronification", Group B versus Group A women showed a statistically significantly higher rate of depression (27/62 corresponding to $43.5 \%$ vs. $26 / 195$ corresponding to $13.3 \%, p<0.01)$ and arterial hypertension (24/62 corresponding to $38.7 \%$ vs. $35 / 195$ corresponding to $17.9 \%, p<0.01)$.

No statistically significant differences were found between the two groups in the presence of other diseases (head injury, hypo- or hyper-thyroidism, colitis, allergy, insomnia, anxiety, and panic attack disorder), either at the time of the first observation or later.

Table 1 Number of days of migraine per month at the first visit

\begin{tabular}{lrll}
\hline & $\begin{array}{l}\leq 5 \text { days per } \\
\text { month }\end{array}$ & $\begin{array}{l}\text { 6 to 10 days per } \\
\text { month }\end{array}$ & $\begin{array}{l}11 \text { to 15 days per } \\
\text { month }\end{array}$ \\
\hline Group A & & & \\
M & $20(41.7 \%)$ & $19(39.6 \%)$ & $7(14.6 \%)$ \\
F & $83(42.6 \%)$ & $59(30.3 \%)$ & $44(22.6 \%)$ \\
Total & $103(42.4 \%)$ & $78(32.1 \%)$ & $51(21.0 \%)$ \\
Group B & & & \\
M & $1(10.0 \%)$ & $3(30.0 \%)$ & $5(50.0 \%)$ \\
F & $20(32.3 \%)$ & $15(24.2 \%)$ & $24(38.7 \%)$ \\
Total & $21(29.2 \%)$ & $18(25.0 \%)$ & $29(40.3 \%)$ \\
\hline
\end{tabular}

11-15 days of MO per month versus $\leq 10$ days of MO per month: Group A versus Group B: $p<0.01$ for the total sample; $p<0.05$ for both men and women 


\section{Conclusions}

Our data analysis indicates that a high number of MO days per month, comorbidity with depression and susceptibility to arterial hypertension in women represent unfavourable prognostic factors for a possible "chronification" of MO. Therefore, it is extremely important that the presence of these elements in all MO subjects be investigated with the utmost care. If present, they should be managed in the most appropriate way in order to prevent migraine from turning chronic.

Conflict of interest None.

\section{References}

1. Headache Classification Subcommittee of the International Headache Society (2004) The International Classification of Headache Disorders. Cephalalgia 24(suppl 1):1-160

2. Olesen J, Bousser MG, Diener HC, Dodick D, First M, Goadsby PJ, Goebel H, Lainez MJA, Lance JW, Lipton RB, Nappi G, Sakai F, Schoenen J, Silberstein SD, Steiner TJ (2006) New appendix criteria open for a broader concept of chronic migraine. Cephalalgia 26:742-746 